\title{
Malacia, inflammation and bronchoalveolar lavage culture in children with persistent respiratory symptoms
}

\author{
F. De Baets*,+, I. De Schutter ${ }^{\#,+}$, C. Aarts ${ }^{\ddagger}$, F. Haerynck*, S. Van daele*, \\ E. De Wachter ${ }^{\#}$, A. Malfroot ${ }^{\#}$ and P. Schelstraete*
}

ABSTRACT: In children with persistent respiratory symptoms despite regular anti-asthma inhalation treatment, diagnostic investigations to exclude underlying disease are warranted.

124 children were prospectively enrolled, and 24-h oesophageal pH measurement and fibreoptic bronchoscopy with bronchoalveolar lavage (BAL) were performed. BAL fluid (BALF) was processed for neutrophil counting and bacterial culture. Inflammation of the respiratory mucosa was assessed.

A structural abnormality of the central airways was found in $47 \%$ of subjects ( $40 \%$ females). In $19 \%$ of subjects, neither anatomical anomalies nor inflamed respiratory mucosa were observed, whereas in $64 \%$, definite macroscopic mucosal inflammation was observed. Inflammation of the respiratory mucosa was associated with a significantly higher percentage of neutrophils in the BALF: median (interquartile range) $48(14-82) \%$ compared with $7(0-16) \%(p<0.025)$. A positive BALF culture was found in $62 \%$ of the infants with mucosal inflammation compared with $25 \%$ in the group without inflammation $(p<0.016) .56 \%$ of the BALF samples were positive for bacterial culture.

In children with persistent respiratory symptoms, nearly half have anatomical anomalies of the central airways. In $62 \%$ of the children with mucosal inflammation, a positive BAL culture and a significantly higher percentage of BALF neutrophils were detected.

KEYWORDS: Bronchitis, infants, laryngomalacia, tracheomalacia, wet cough, wheezing

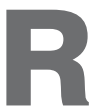
espiratory symptoms, such as productive cough, bronchorrhoea and wheezing, are common problems in infants and young children. Prevalence of up to $33 \%$ has been reported [1]. A diagnosis of asthma at that age is difficult, and relies on the clinical and familial history. Assessment of young children with recurrent wheeze or cough is difficult in the clinical setting due to a relative lack of objective physiological measures and diagnostic tools. Consequently, it is common practice to initiate empirical trials of antiasthma medications and use symptom responses as a diagnostic tool. The European Respiratory Society recommends selectively referring young children who do not respond to such medical interventions for flexible bronchoscopy with bronchoalveolar lavage (BAL) to evaluate other aetiologies of chronic/ recurrent wheeze and cough $[2,3]$. In a retrospective study, SCHELLHASE et al. [4] reported airway anomalies in $57 \%$ of 30 children, 0-18 months of age, with recurrent episodes of wheezing that were clinically refractory or poorly responsive to inhaled albuterol. SAITO et al. [5] reported a high percentage of positive BAL fluid (BALF) cultures in children with recurrent cough and/or wheeze who had failed to respond to empirical treatment for asthma and gastro-oesophageal reflux.

We initiated a prospective study incorporating fibreoptic bronchoscopy (FOB) and BAL, a sweat test, and 24-h oesophageal $\mathrm{pH}$ measurement in children with treatment-resistant respiratory symptoms.

\section{METHODS}

Patients were recruited in the paediatric pulmonary departments of Ghent University Hospital (Ghent, Belgium) and the Universitair Ziekenhuis Brussel (Brussels, Belgium) between January 2006 and August 2008. Infants and young children aged 02 yrs who had persistent respiratory symptoms, productive cough, bronchorrhoea and wheezing for $\geqslant 3$ months, and had been sent by their paediatrician for advice were evaluated. They received inhalation treatment via spacer (fluticasone dipropionate; $100 \mu \mathrm{g}$ twice a day) or aerosol (budesonide; $250 \mu \mathrm{g}$ twice a day) combined with a $\beta$-2-agonist (salbutamol; $100 \mu \mathrm{g}$ twice a day) during the first week, for $\geqslant 1$ month. Patients unresponsive to adequate asthma treatment, with symptoms defined as ongoing or
AFFILIATIONS

*Dept of Pediatric Pulmonology, Ghent University Hospital, Ghent, \#Dept of Pediatrics, Paediatric Pulmonology, CF Clinic and Pediatric Infectious Diseases, Universitair Ziekenhuis Brussel, Brussels, Belgium.

-Dept of Pediatric Pulmonology

Radboud University Nijmegen

Medical Centre, Nijmegen, The Netherlands.

+These authors contributed equally to the study.

\section{CORRESPONDENCE}

F. De Baets

Dept of Pediatric Pulmonology

Ghent University Hospital

De Pintelaan 185

9000 Gent

Belgium

E-mail: frans.debaets@ugent.be

Received:

Feb 232011

Accepted after revision:

May 052011

First published online:

July 202011

European Respiratory Journal

Print ISSN 0903-1936

Online ISSN 1399-3003 
recurrent and who had not undergone antibiotic treatment in the previous month were enrolled. Treatment compliance was inquired about but not verified.

Children born prematurely or showing failure to thrive, children with a positive sweat test, prolonged endotracheal intubation or tracheotomy, dysmorphic children, and children with congenital cardiopathies (frequently associated with primary tracheomalacia) or neurological disorders were excluded [6].

Children with localised consolidations on a chest radiography were excluded and treated with an antibiotic.

In the course of a 2.5 -yr period, 124 children, representing $2.4 \%$ of all children between 0 and 2 yrs of age evaluated for sustained respiratory disorders at our centre during this period, were enrolled. Family history of allergy and asthma in first-degree relatives was positive in 39 and $17 \%$ of the study patients, respectively. $14 \%$ of the children had been chronically exposed to cigarette smoke.

A 24-h oesophageal $\mathrm{pH}$ measurement was performed in all patients (DL70; Ecce Medical, Schoten, Belgium). A positive test was defined based on gastroenterological criteria: oesophageal $\mathrm{pH}<4$ for $>4 \%$ of the measuring time; and $>25$ reflux moments and at least one reflux period $>5 \mathrm{~min}$ [7].

All recruited patients underwent FOB (BF-3C20; Olympus, Hamburg, Germany). FOB was performed using the nasal approach under sedation and local anaesthesia in a spontaneously breathing child. The BAL was performed in the right lower lobe by instillation and aspiration of three aliquots of $1 \mathrm{~mL} \cdot \mathrm{kg}^{-1}$ body weight of normal saline. BALF recovered after each instillation was gathered in different sterile receptacles. The third BALF sample was used for bacterial culture and white blood cell differentiation. Cell pellets were made by cytospin. A smear of the cytospin was stained with Giemsa for white blood cell differentiation.

The slides were examined using light microscopy by three independent observers, who were blinded to clinical status and bronchoscopic findings. The mean percentage of the three observations was reported.

In order to isolate Gram-positive bacteria, Gram-negative bacteria and Staphylococcus aureus, BALF was cultured on blood agar, MacConkey agar and mannitol salt agar, respectively. Morphologically different colonies were picked for further identification. Every potential respiratory pathogen was reported; commensal organisms were disregarded.

Laryngomalacia was defined as a dynamic anomaly of the supraglottis region causing stridor by narrowing of the laryngeal entry, and tracheomalacia and bronchomalacia as an abnormal collapse $(>50 \%)$ of the trachea or the main bronchi, respectively, as estimated by the bronchoscopist, due to localised or generalised weakness of the airway wall, leading to respiratory obstruction [6].

Inflammation of the respiratory mucosa was defined as mucosal oedema and/or hyperaemia, and/or hypertrophic submucosal glands (cobble-stone pattern), and/or longitudinal mucosal folds.

In children with localised pulsatile tracheomalacia, angiographic magnetic resonance imaging (MRI) was performed in order to detect vascular anomalies.
The study was approved by the ethical committees of both participating hospitals. Written informed consent was obtained from both parents.

\section{Statistics}

Statistical significance of differences between subgroups for prevalence of mucosal inflammation, allergy and asthma in firstdegree relatives, as well as passive smoking, were calculated using the Chi-squared test. The nonparametric Wilcoxon test was used for calculation of statistical significance of differences in granulocyte count between patients with and without respiratory mucosal inflammation. A p-value $<0.05$ was considered significant.

\section{RESULTS}

We selected 124 children (58 females; median age 10 months, interquartile range (IQR) 7-14 months) with treatment-resistant respiratory symptoms for additional diagnostic work-up. A 24-h oesophageal $\mathrm{pH}$ measurement was positive in $29 \%$ of the children. In 20 children, the 24-h oesophageal $\mathrm{pH}$ measurement was not conclusive because of digital storage problems. A structural abnormality of the central airways was found in $46 \%$ (58 out of 124 ) subjects ( $40 \%$ female; 23 out of 58 ): $6 \%$ (seven out of 124) laryngomalacia, $19 \%$ (24 out of 124 ) tracheomalacia, $10 \%$ (12 out of 124) combined laryngotracheomalacia, 9\% (11 out of 124) bronchomalacia and 3\% (four out of 124) localised midtracheal pulsatile compression (fig. 1). In the four infants with localised pulsatile compression, vascular MRI was conclusive for innominate artery syndrome.

In 24 children, neither anatomical anomalies nor inflammation of the respiratory mucosa were observed, whereas in 79 , definite mucosal inflammation was reported. Mucosal inflammation was found in $64 \%$ (30 out of 47 ) of the children with tracheomalacia and/or bronchomalacia. In children with inflammation of the respiratory mucosa, a significantly higher percentage of BALF polymorphonuclear leukocytes was found: $48 \%$ (IQR 14-82\%) compared with $7 \%$ (IQR $0-16 \%$ ) in the noninflammation group $(\mathrm{p}<0.025)$ (fig 2).

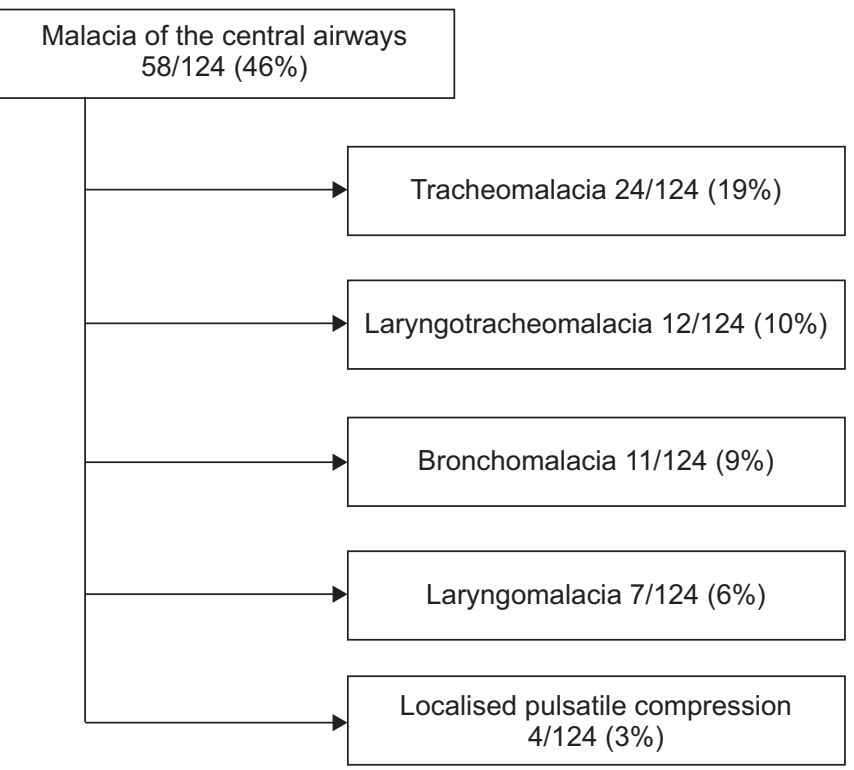

FIGURE 1. Relative prevalence of different types of airway malacia. 


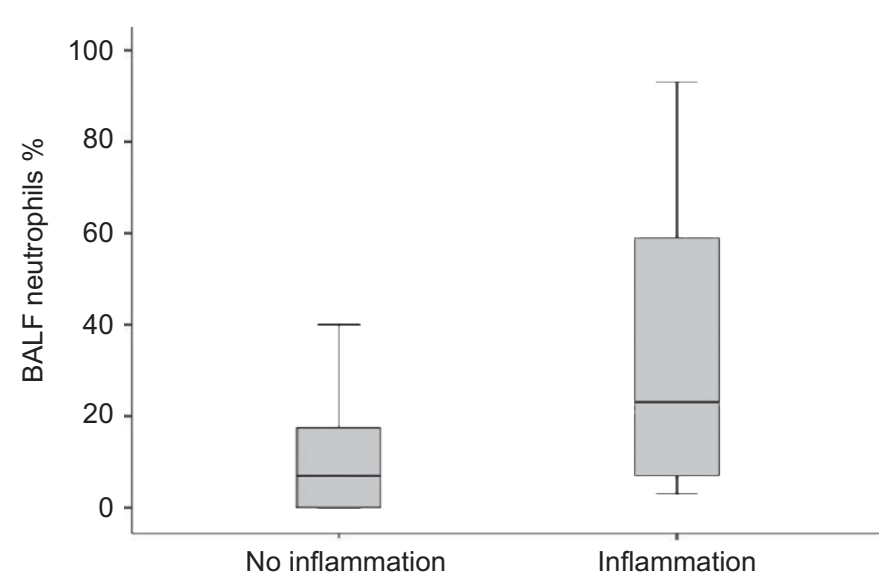

FIGURE 2. Percentage of neutrophils in bronchoalveolar lavage (BAL) of infants without and with inflammation of the respiratory mucosa. $p<0.025$.

In $62 \%$ (49 out of 79) of the children with mucosal inflammation, a positive BALF culture was obtained compared to $25 \%$ (six out of $24)$ in the noninflammation group $(p<0.016)$ (fig 3$) .56 \%$ of the BALF samples were positive for bacterial culture: $51 \%$ Moraxella catharralis, 28\% Haemophilus influenzae, 13\% Streptococcus pneumoniae, 10\% Staphylococcus aureus, 4\% Escherichia coli, 1\% Klebsiella oxytoca and $1 \%$ Pseudomonas aeruginosa. In patients with a positive BALF culture, a higher median value of polymorphonuclear cells was found. Because of the large range, however, this finding is not significant. Regarding gastro-oesophageal reflux, no significant difference was found in positive bacterial cultures between children with a positive or a negative test: $65 \%$ (19 out of 29) compared with $55 \%$ (41 out of 75 ).

Compared with the group with normal anatomy of the conducting airways, patients with tracheo- and bronchomalacia showed a significantly lower percentage of positive familial history of asthma in first relatives (15 versus $24 \% ; \mathrm{p}<0.016)$ and passive smoking ( 0 versus $18 \% ; \mathrm{p}<0.012)$. No significant difference in allergy in first-degree relatives was observed (38 versus $43 \%$; nonsignificant).

\section{DISCUSSION}

We report high prevalence of anatomical anomalies of the central airways in children with treatment-resistant respiratory symptoms: laryngomalacia, tracheomalacia, laryngotracheomalacia and bronchomalacia were observed in $46 \%$ of subjects.

In a retrospective study, SCHELLHASE et al. [4] reported central airway anomalies in $57 \%$ of 30 children, $0-18$ months of age, with recurrent episodes of wheezing that were clinically refractory or poorly responsive to inhaled albuterol. Central airway abnormalities were found in 17 (57\%) subjects and tended to be more common in the 0-6-month age group. They found comparable prevalence of total anomalies (57\%) and laryngomalacia (6\%) but a higher frequency of segmental tracheomalacia (40\%), caused by vascular anomalies in 33\% of subjects. This high prevalence of vascular compression may be due to the bias of the retrospective design of the study. SAGLANI et al. [8] studied the clinical benefit of further investigations in preschool children with severe recurrent wheeze. Noisy breathing with or without wheezing periods was the main inclusion criterion. All patients had already failed to

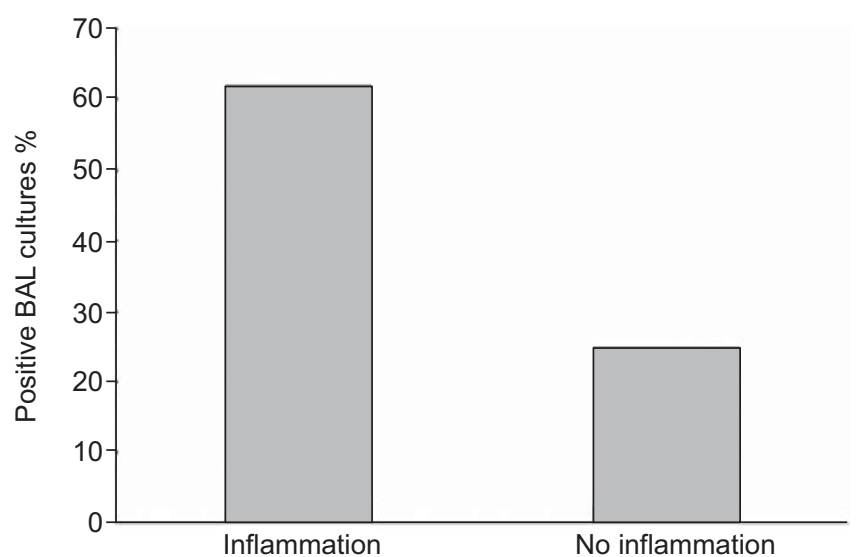

FIGURE 3. Percentage of positive bronchoalveolar lavage fluid (BALF) cultures in infants with and without chronic inflammation of the respiratory mucosa $p<0.016$.

respond to bronchodilator or to (inhaled or oral) steroid therapy. The minimum duration of the inhaled steroid trial was 2 months. In a subgroup of 19 young children ( $<18$ months of age), structural anomalies of the central airways were reported in $53 \%$. No further information on specific anomalies was given and children with enlarged tonsils and adenoids were also included.

SAITO et al. [5] studied 19 infants with recurrent wheeze and cough, and reported tracheomalacia in $63 \%$ of subjects.

Despite subtle differences in inclusion criteria (recurrent wheezing poorly responsive to albuterol [3], noisy breathing with or without wheezing poorly responsive to $\beta$-agonists and inhaled steroids [8], and recurrent wheeze and cough [5]), our prospective study of a large group of children revealed comparative prevalences of central airway abnormalities in children with treatment-resistant respiratory symptoms of productive cough, bronchorrhoea and wheezing. This finding corroborates the high prevalence of central airway anomalies in young children with treatment-resistant cough and/or noisy breathing with or without wheezing, and justifies the referral for bronchoscopy.

Primary or congenital tracheobronchomalacia is seen in normal and prematurely born infants, congenital anomalies of the cartilage, and congenital syndromes [6]. According to BOOGAARD et al. [9], the estimated prevalence of primary tracheo- and or bronchomalacia amounted to one in 2,100 children aged 0-17 yrs. In infants, CALLAHAN [10] estimated the prevalence of congenital tracheomalacia at one in 1,445. Secondary or acquired tracheo- and bronchomalacia are caused by prolonged intubation, tracheotomy, severe tracheobronchitis or compression. No data are available concerning the prevalence of acquired tracheobronchomalacia. In our study population, tracheobronchomalacia was closely associated with mucosal inflammation. Although, an association does not prove a causal origin and our study was not designed to answer the question of what came first, one could speculate that malacia in this kind of patient is sustained and could even be induced by ongoing mucosal inflammation [6].

In $64 \%$ of the study group, definite macroscopic inflammation of the respiratory mucosa was observed. A significantly higher percentage of polymorphonuclear cells was found in this subgroup of children, corroborating the finding of inflammation 
of the respiratory mucosa. $64 \%$ of the children with tracheobronchomalacia also displayed signs of mucosal inflammation. SAITO et al. [5] reported visible inflammation of the respiratory mucosa in $42 \%$ of their study group and an increase of neutrophils in the BALF (>10\%) in $47 \%$.

In the children with mucosal inflammation, we found a significant higher percentage of positive bacterial BALF cultures. Moreover, in these children, as a characteristic of infection, a significantly higher percentage of polymorphonuclear cells was counted. These findings are suggestive of protracted bacterial infection as a possible aetiological, or at least concomitant, cause of persistent respiratory symptoms. SCHELLHASE et al. [4] reported, in 27 infants in whom BAL was performed, that three $(11 \%)$ had a positive bacterial culture (H. influenzae and $M$. catharralis). In a retrospective study, DONNELLY et al. [11] reported the possibility of persistent bacterial bronchitis in children with chronic wet cough diagnosed as asthma. Prolonged and sometimes recurrent treatment with antibiotics led to recovery in over half of these patients. They suggested that this treatment might prevent an evolution to bronchiectasis. In $56 \%$ of our study group, a positive bacterial culture was obtained, mimicking the bacterial flora found in sputum of children with bronchiectasis.

SAGLANI et al. [8] identified bacterial bronchitis in $43 \%$ of children with persistent wheeze, despite adequate treatment for asthma ( $24 \%$ were receiving oral steroids).

SAITO et al. [5] reported positive bacterial BALF cultures in $61 \%$ of their study group. Those authors stated that aspiration is likely to be an important predisposing condition in early childhood leading to disruption of the normal epithelium and colonisation by organisms such as H. influenzae. Strengthening this hypothesis, we found, in a recent study, that aspiration was a major problem in $48 \%$ of children with laryngotracheomalacia and persistent respiratory symptoms [12].

In view of the widely accepted "vicious circle" hypothesis that the development of bronchiectasis evident on high-resolution computed tomography is preceded by a period of chronic inflammation driven by persistent bacterial infection of the conducting airways, the high percentage of positive BAL cultures in our study group is important and worrying [13, 14]. Double-blind, placebocontrolled trials are warranted to study the immediate and delayed effects of antibiotic treatment in this small subgroup of patients.

Our study was the first to prospectively enrol a large group of infants with treatment-resistant respiratory symptoms of productive cough, bronchorrhoea and wheeze. The high prevalence of positive BALF cultures corroborated the results of smaller retrospective studies.

These observations emphasise the need for FOB and BAL in children with ongoing respiratory symptoms, productive cough, bronchorrhoea and wheezing, despite an adequate anti-asthma inhalation treatment $[2,3]$. A diagnosis of malacia could explain to the parents the persistent character of the respiratory problems and point to additive treatment options, such as physiotherapeutic positive end-expiratory pressure techniques. In the case of mucosal inflammation and a positive bacterial BALF culture, a prolonged therapeutic trial with an antibiotic could help to control the respiratory symptoms and probably prevent an evolution to the development of bronchiectasis [11].
The present study has some weaknesses: mucosal inflammation was not proved by biopsy and BALF cultures were not performed in a quantitative way. The significant higher percentage of neutrophils in the BALF of patients with macroscopic mucosal inflammation could preclude this criticism. In order to preclude upper airway-induced positive BAL cultures, samples were taken out of the third instillation. Moreover, significantly more positive cultures were seen in the patient group with mucosal inflammation.

High prevalence of central-airway anomalies and mucosal inflammation, high percentages of neutrophils, and positive BALF cultures are observed in young children with treatmentresistant respiratory symptoms. Our study emphasises the need for further investigations to elucidate the role of each of these findings in the pathogenesis of treatment-resistant respiratory symptoms in young children.

\section{STATEMENT OF INTEREST}

None declared.

\section{ACKNOWLEDGEMENTS}

The authors thank M. Remi and S. Kimpe for their technical assistance with bronchoscopy, and L. De Baets (all Dept of Pediatric Pulmonology, Ghent University Hospital, Ghent, Belgium) for English language review.

\section{REFERENCES}

1 Brand PL, Baraldi E, Bisgaard H, et al. Definition, assessment and treatment of wheezing disorders in preschool children: an evidence-based approach. Eur Respir J 2008; 32: 1096-1110.

2 Midulla F, de Blic J, Barbato A, et al. Flexible endoscopy of paediatric airways. Eur Respir J 2003; 22: 698-708.

3 De Blic J, Midulla F, Barbato A, et al. Bronchoalveolar lavage in children. ERS Task Force on Bronchoalveolar Lavage in Children. Eur Respir J 2000; 15: 217-231.

4 Schellhase DE, Fawcett DD, Schutze GE, et al. Clinical utility of flexible bronchoscopy and bronchoalveolar lavage in young children with recurrent wheezing. J Pediatr 1998; 132: 312-318.

5 Saito J, Harris WT, Gelfond J, et al. Physiologic, bronchoscopic, and bronchoalveolar lavage fluid findings in young children with recurrent wheeze and cough. Pediatr Pulmonol 2006; 41: 709-719.

6 Carden KA, Boiselle PM, Waltz DA, et al. Tracheomalacia and tracheobronchomalacia in children and adults: an in-depth review. Chest 2005; 127: 984-1005.

7 Rudolph CD, Mazur LJ, Liptak GS, et al. Guidelines for evaluation and treatment of gastroesophageal reflux in infants and children: recommendations of the North American Society for Pediatric Gastroenterology and Nutrition. J Pediatr Gastroenterol Nutr 2001; 32: Suppl. 2, S1-S31.

8 Saglani S, Nicholson AG, Scallan M, et al. Investigation of young children with severe recurrent wheeze: any clinical benefit? Eur Respir J 2006; 27: 29-35.

9 Boogaard R, Huijsmans SH, Pijnenburg MWH, et al. Tracheomalacia and bronchomalacia in children. Chest 2005; 128: 3391-3397.

10 Callahan CW. Primary tracheomalacia and gastroesophageal reflux in infants with cough. Clin Pediatr 1998; 37: 725-731.

11 Donnelly D, Critchlow A, Everard ML. Outcomes in children treated for persistent bacterial bronchitis. Thorax 2007; 62: 80-84.

12 De Baets F, Aarts C, Van daele S, et al. Milk protein and oil-red-O staining of alveolar macrophages in chronic respiratory disease of infancy. Pediatr Pulmonol 2010; 45: 1213-1219.

13 Cole PJ, Wilson R. Host-microbial interrelationships in respiratory infection. Chest 1989; 95: Suppl. 3, 217S-221S.

14 Cole P. The damaging role of bacteria in chronic lung infection. J Antimicrob Chemother 1997; 40: 5-10. 